\title{
Evaluation of Fuzzy Inference System in Image Processing
}

\author{
Jaideep Kaur \\ Department of ECE \\ DAVIET Jalandhar
}

\author{
Poonam Sethi \\ Department of ECE \\ DAVIET Jalandhar
}

\begin{abstract}
This paper reports the evaluation of Fuzzy logic implemented on image, in MATLAB environment, used to implement different functions. In this paper, the in-built functions of MATLAB are implemented on image and the resultant images are displayed using different functions. Fuzzy inference system has been designed for four inputs, one output that tells whether the pixel under consideration is "low", "medium" or "high" pixel. Rule base comprises of eight rules, which classify the target pixel. The Type-1 Fuzzy Logic System Toolbox (T1FLS) is an environment for type-1 fuzzy logic inference system development. Tools that cover the different phases of the fuzzy system design process, from the initial description phase, to the final implementation phase, build the Toolbox. The Toolbox's best qualities are the capacity to develop complex systems and the flexibility that permits the user to extend the availability of functions for working with the use of type-1 fuzzy operators, fuzzy variables, type-1 membership functions, de-fuzzification methods and the evaluation of Type-1 Fuzzy Inference Systems.
\end{abstract}

\section{Keywords}

Fuzzy Logic System, Fuzzy Inference System, Edge detection, Digital image processing, T1FIS.

\section{INTRODUCTION}

In the area of digital signal processing, methods have been proven that solve the problem of image processing. Edge detection is a terminology used particularly in the areas of feature extraction, to refer to algorithms which aim at identifying points in a digital image at which the image brightness changes sharply or more formally has discontinuities [2]. The goal of edge detection is to locate the pixels in the image that correspond to the edges of the objects seen in the image. This is usually done with a first and/or second derivative measurement following by a comparison with threshold which marks the pixel as either belonging to an edge or not. The result is a binary image which contains only the detected edge pixels.

The fuzzy sets were presented by L.A. Zadeh in 1965 [2, 3] to process or manipulate data and information affected by unprobabilistic, uncertainty or imprecision. These were designed to mathematically represent the vagueness and uncertainty of linguistic problems and considered as a generalization of the classic set theory. The work of Fuzzy image processing is the beginning of an effort for the design of new pre-processing images techniques, using Fuzzy Inference System (FIS), which allow feature extraction [1, 7]. Based on this technology, firstly set the image fuzzy characteristic plane of original image, secondly precede the fuzzy enhancement, and then detect the edge by sobel differential arithmetic [4]. In the past decade, fuzzy systems have replaced conventional technology in different scientific and system engineering applications, especially in pattern recognition and control systems [2]. The work of this paper is concerned with the development of fuzzy logic rules based algorithm for the detection of image edges. In the proposed technique, it is necessary to apply Fuzzy Inference System to generate the vector of edges [5].

\section{FUZZY INFERENCE SYSTEM}

Fuzzy inference is the process of formulating the mapping from a given input to an output using fuzzy logic. The mapping then provides a basis from which decisions can be made, or patterns discerned. The process of fuzzy inference involves all of the pieces related to membership functions, fuzzy logic operators, and if-then rules [10]. There are two types of fuzzy inference systems that can be implemented in the Fuzzy Logic Toolbox: Mamdani type and Sugeno-type. These two types of inference systems vary somewhat in the way outputs are determined. Fuzzy inference systems have been successfully applied in fields such as automatic control, data classification, decision analysis, expert systems, and computer vision. Because of its multidisciplinary nature, fuzzy inference systems are associated with a number of names, such as fuzzy-rule-based systems, fuzzy expert systems, fuzzy modeling, fuzzy associative memory, fuzzy logic controllers, and simply fuzzy systems. The Fuzzy inference system in MATLAB works as per shown below (see Figure 1):

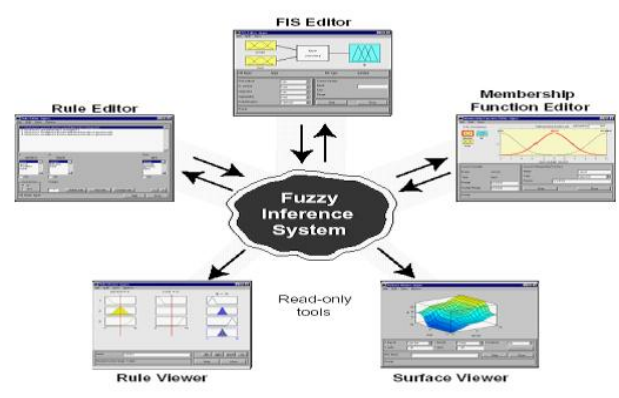

Figure 1

\subsection{Design and Implementation}

The following are the steps which are carried out in the sequence to get the desired output:

1) Read the original image.

2) Convert it into gray plane.

3) Calculate size of original image.

4) Apply Fuzzy Inference Engg.

5) Passing parameters to the FIS 


\subsection{Edge Detection with Type-1 Fuzzy Logic}

A Mamdani FIS has been implemented using T1FLS, with four inputs, one output and 7 rules, using the MATLAB Fuzzy Logic ToolBox 7 [6].

\subsection{Inputs for Type-1 FIS}

For the Fuzzy Inference System Type-1, 4 inputs are required, 2 of them are the gradients with respect to $\mathrm{x}$-axis and y-axis, calculated with (1) and (2), to which we will call DH and DV respectively.

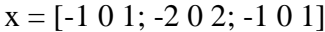

gx $=$ conv2(double('mai.png'),x,'same') -(1)

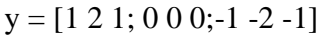

gy=conv2(double('mai.png'),y,'same') -(2)

The other two inputs are filters that calculate two masks by convolution to the original image when applied. One is a high-pass filter, given by the mask of the equation (3), and the other is a low-pass filter given by the mask of equation (4). The high-pass filter hHP detects the contrast of the image to guarantee the border detection in relatively low contrast regions. The low-pass filter hMF allows to detect image pixels belonging to regions of the input where the mean gray level is lower. The goal here is to design a system which makes it easier to include edges in low contrast regions, but which does not favour false edges by effect of noise [7].

$$
\begin{aligned}
& \mathrm{hHP}=\left[\begin{array}{rrr}
-\frac{1}{16} & -\frac{1}{8} & -\frac{1}{16} \\
-\frac{1}{8} & \frac{3}{4} & -\frac{1}{8} \\
-\frac{1}{16} & -\frac{1}{8} & -\frac{1}{16}
\end{array}\right] \\
& \mathrm{hMF}=\frac{1}{25} *\left[\begin{array}{ccccc}
1 & 1 & 1 & 1 & 1 \\
1 & 1 & 1 & 1 & 1 \\
1 & 1 & 1 & 1 & 1 \\
1 & 1 & 1 & 1 & 1 \\
1 & 1 & 1 & 1 & 1
\end{array}\right]
\end{aligned}
$$

Then the inputs for FIS type 1 are: $\mathrm{DH}=\mathrm{gx}, \mathrm{DV}=\mathrm{gy}, \mathrm{HP}=\mathrm{hHP} * \mathrm{I}, \mathrm{M}=\mathrm{hMF} * \mathrm{I}$

Where $*$ is the convolution operator.

\subsection{Fuzzy variables}

For all the fuzzy variables, the membership functions are Gaussian. According to the tests performed the values in DH and DV, go from -800 to 800 , then the ranks in $\mathrm{x}$-axis are adjusted (see Figure 2, 3 \& 4), where the membership functions are:

LOW: gaussmf $(43,0)$,

MEDIUM: gaussmf $(43,127)$

HIGH: gaussmf( 43,255$)$.

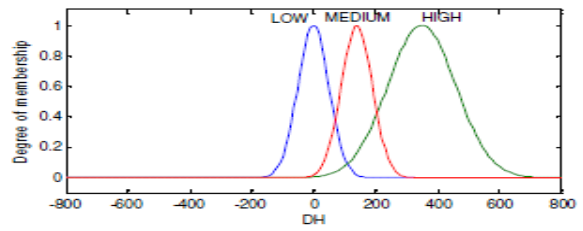

Figure 2 Input variable DH

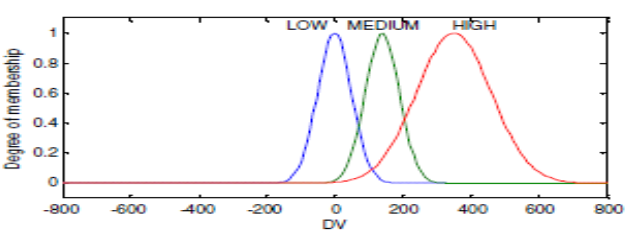

Figure 3 Input variable DV

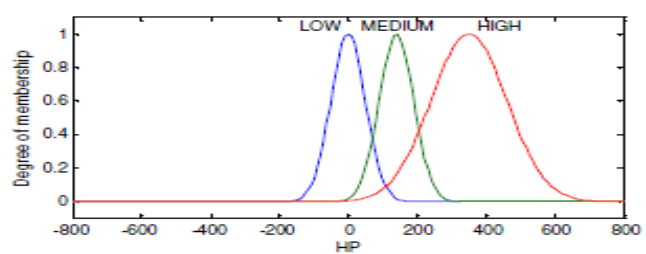

Figure 4 Input variable HP

In the case of variable $M$, the tests use the values in the rank from 0 to 255, and thus the rank in $\mathrm{x}$-axis is adjusted (see Figure 5)

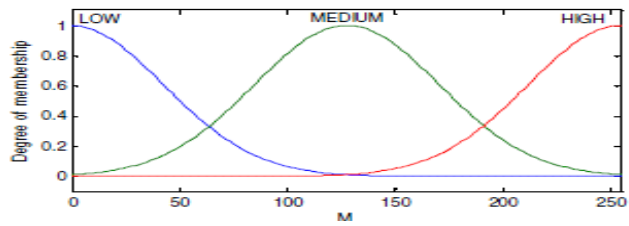

Figure 5 Input variable $\mathrm{M}$

The output variable is EDGES that also adjusted the ranks between 0 and 255 (see Figure 6), as it is the range of values required to display the edges of an image.

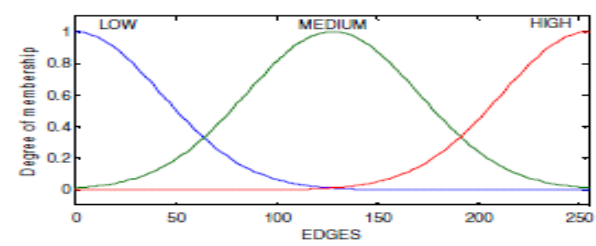

Figure 6 Output variable EDGES

\subsection{Fuzzy Editor}

Figure $7 \& 8$ show the main viewport of the Type-1 Fuzzy Inference Systems Plot and Editor. The FIS Editor displays general information about a fuzzy inference system. There's a simple diagram at the top that shows the names of each input variable on the left, and those of each output variable on the right. The sample membership functions shown in the boxes are just icons and do not depict the actual shapes of the membership functions $[9,10]$.

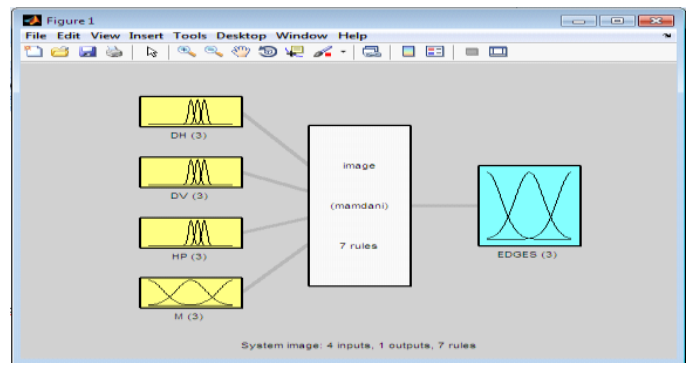

Figure 7 plotfis 


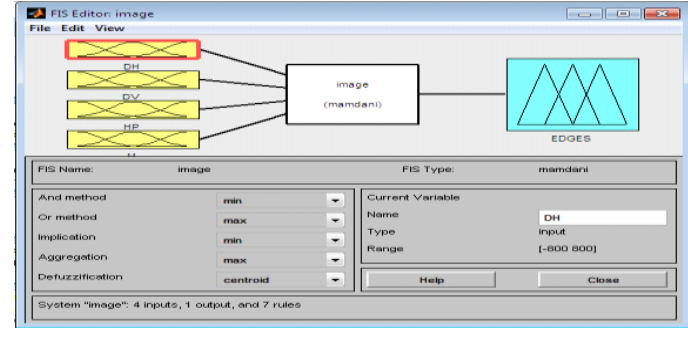

Figure 8 fis editor

\subsection{Fuzzy Inference Rules}

The seven fuzzy rules that allow to evaluate the input variables, so that the exit image displays the edges of the image [5,7].

1. If (DH is LOW) and (DV is LOW) then (EDGES is LOW)

2. If (DH is MEDIUM) and (DV is MEDIUM) then (EDGES is HIGH)

3. If (DH is HIGH) and (DV is HIGH) then (EDGES is $\mathrm{HIGH})$

4. If (DH is MEDIUM) and (HP is LOW) then (EDGES is HIGH)

5. If (DV is MEDIUM) and (HP is LOW) then (EDGES is HIGH)

6. If ( $\mathrm{M}$ is LOW) and (DV is MEDIUM) then (EDGES is LOW)

7. If ( $\mathrm{M}$ is LOW) and (DH is MEDIUM) then (EDGES is LOW)

\subsection{Fuzzy Type-1 FIS Rule Editor}

Figure $9 \& 10$ show the FIS rule editors of the T1FIS, Rule edit and Rule view [8]. Constructing rules using the graphical Rule Editor interface is fairly self-evident. Based on the descriptions of the input and output variables defined with the FIS Editor, the Rule Editor allows us to construct the rule statements automatically, by clicking on and selecting one item in each input variable box, one item in each output box, and one connection item. Rules may be changed, deleted, or added, by clicking on the appropriate button.

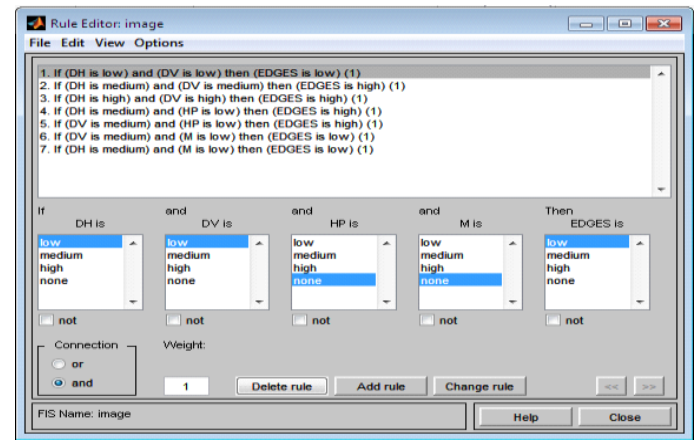

Figure 9 rule editor

The Rule Viewer displays a roadmap of the whole fuzzy inference process. It's based on the fuzzy inference diagram described in the previous section. A single figure window with 7 small plots nested in it (see Figure 10). The three small plots across the top of the figure represent the antecedent and consequent of the first rule. Each rule is a row of plots, and each column is a variable. The first two columns of plots (the yellow plots) show the membership functions referenced by the antecedent, or the if-part of each rule. The last column of plots (the blue plots) shows the membership functions referenced by the consequent, or the then-part of each rule [6].

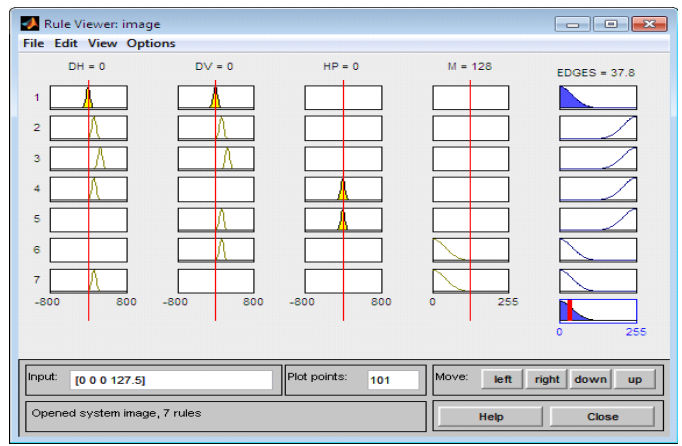

Figure 10 rule viewer

\subsection{Fuzzy Type-1 FIS Surface Editor}

Figure 11 show the FIS surface editor of the Type-1 Fuzzy Inference Systems, namely surf-view. Upon opening the Surface Viewer, we are presented with a two-dimensional curve that represents the mapping from service quality to tip amount. Since this is a four-input one-output case, we can see the entire mapping in different plots. Two-input and oneoutput systems work well, as they generate three-dimensional plots [11].

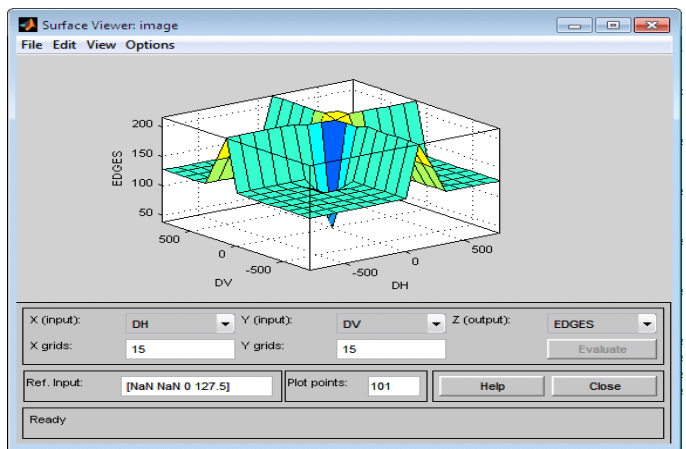

Figure 11 surfview

\section{FIS Type 1 FUZZY IMAGE PROCESSING RESULTS}

Reviewing the values of each pixel, we see that all fall in the rank from 0 to 255 , which is not obtained with the method of gradient magnitude.

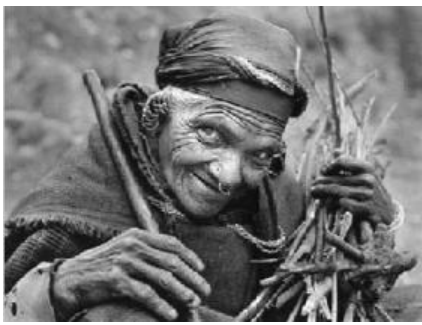

Figure 12 Original Input image 


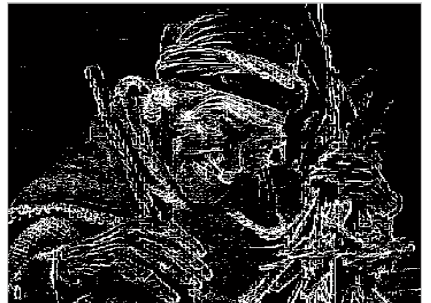

Figure 13 EDGES image by Fuzzy Type-1 with black background and with adjusted threshold

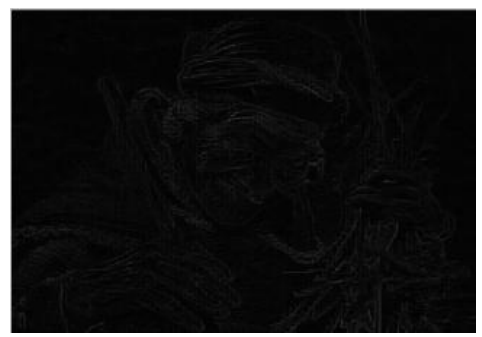

Figure 14 EDGES image by Fuzzy Type-1 with black background

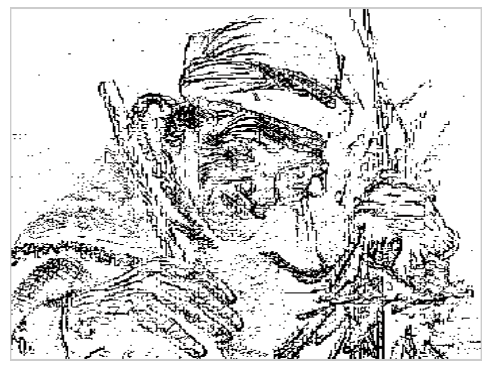

Figure 15 EDGES image by FISTYPE-1 with white background and with adjusted threshold

\section{CONCLUSION}

The result was obtained by the Type-1 Fuzzy Inference System, because it was possible to clear more than half of the pixels without depreciating the image. An important advantage of the Fuzzy Inference Systems is that diverse variables can be modeled as noise and contrast. This causes that the results are near to the reality and therefore they are more reliable.

\section{REFERENCES}

[1] L. R. Palafox-Maestre, (Tijuana México, Mayo del 2002), "Técnicas de Procesamiento de Imágenes Utilizando un Procesador Digital de Señales". Centro de Investigación y Desarrollo de Tecnología Digital.

[2] Zadeh, L.A., "Fuzzy sets," Information and Control, Vol. 8, pp. 338-353, 1965

[3] Zadeh, L.A., "Outline of a new approach to the analysis of complex systems and decision processes," IEEE Transactions on Systems, Man, and Cybernetics, Vol. 3, No. 1, pp. 28-44, Jan. 1973.

[4] M.D. Heath, (Florida, Mayo de 1996), "A Robust Visual Method for Assessing the Relative Performance of Edge Detection Algorithms", Available: http://marathon.csee.usf.edu/edge/edge_detection.html

[5] Olivia Mendoza, Patricia Melin, Guillermo Licea (2007) " A New Method for Edge Detection in Image Processing using Interval Type-2 Fuzzy Logic" ,IEEE International Conference on Granular Computing )

[6] The MathWorks, Inc., "Fuzzy Logic Toolbox 2.2.4", (Sept. 2006) http://www.mathworks.com/products/matlab/,

[7] C.J. Miosso, A. Bauchspiess, (April, 2001), "Fuzzy Inference System Applied to Edge Detection in Digital Images", V Brazilian Conference on Neural Networks.

[8] J. Mendel, Uncertain Rule-Based Fuzzy Logic Systems: Introduction and New Directions. NJ: Prentice-Hall, 2001.

[9] The MathWorks, Inc.,'Image Processing Toolbox 7.9.0", (August, 2009) http://www.mathworks.com/products/matlab/

[10] M. Nachtegael, D. Van derWeken, and E. E. Kerre, "Fuzzy techniques in image processing: Three case studies," Int. J. Comput. Anticipatory Syst., vol. 12, pp. 89-104, Aug. 2002.

[11] C. Cornelis, G. Deschrijver, and E. E. Kerre, "Classification of intuitionistic fuzzy implicators: An algebraic approch," in Proc. $6^{\text {th }}$ Joint Conf. Information Sciences, 2002, pp. 105-108. 